\title{
From a sector-based service model to a functional one: qualitative study of staff perceptions
}

\section{AIMS AND METHOD}

An increasing number of mental health trusts in the UK are replacing the geographically sectorised model of service delivery with a functional one. We conducted a qualitative study of staff perception concerning this reorganisation in ourTrust, to inform effective change management locally and elsewhere.
RESULTS

Perceived benefits were quick decision-making, positive risk management and improvement in clinical leadership. Dedicated consultant time would lead to improvements in junior doctor training. Concerns included lack of continuity of care, disagreement between teams and de-skilling of staff. Communication was seen as the key to overcoming these difficulties.

CLINICAL IMPLICATIONS

Detailed communication from management about the practicalities and reasons for change could facilitate effective transition.
New Ways of Working is an initiative led by the Royal College of Psychiatrists and the National Institute of Mental Health in England. ${ }^{1}$ It offers a solution to the perceived shortcomings of existing sector-based adult mental health services, along with various other recommendations. It was felt that consultants were stretched between the in-patient and community teams, ${ }^{1}$ burdened by large personal case-loads, ${ }^{2}$ and working long hours with unlimited demands and endless risks. ${ }^{3}$

As part of the New Ways of Working initiative, some mental health trusts piloted replacing the geographically sectorised model with a functional one. ${ }^{1}$ Consultants were allocated to teams, either in the community or based in a hospital. They were no longer responsible for patients across the full range of treatment settings. This is consistent with previous government guidelines that each in-patient ward must have a dedicated lead consultant psychiatrist. ${ }^{4}$ An increasing number of mental health trusts in the UK are now redesigning services in line with these recommendations. ${ }^{5}$

In March 2008, in one locality of our National Health Service (NHS) Foundation Trust, adult mental health services were reorganised from a sector-based model to a functional one. Although these changes came after a period of consultation with staff and other key stakeholders, a significant level of anticipation and anxiety was evident among clinical staff prior to this change. An extensive electronic database search revealed no study aimed at exploring the opinions of multidisciplinary clinical staff. We report a qualitative study of staff perception concerning this reorganisation.

\section{Method}

\section{Setting}

The study took place between January and March 2008 in an adult mental health service in East Anglia covering a population of nearly 200000 .

\section{Participants}

A theoretical sampling method was used to identify members of multidisciplinary teams involved in direct patient care. This included staff from in-patient units, community mental health teams (CMHTs) and specialist teams such as crisis resolution and home treatment, early intervention, assertive outreach and rehabilitation psychiatry. Interviews were carried out until saturation of themes had been reached. In total, 21 people were interviewed (3 consultants, 4 trainee doctors, 3 team leaders and managers, 5 senior nurses, 2 social workers, 2 support treatment and recovery workers, 1 psychologist and 1 occupational therapist). All participants provided informed consent.

\section{Interviews}

A semi-structured interview relating to forthcoming changes was conducted (G.K., S.C. and M.K.R.). Prompts included differences between the current and proposed system, opportunities provided, foreseeable challenges and means of overcoming them. Interviewees were also asked how patients might perceive this change. Each participant was interviewed face to face once, either individually or as part of a focus group facilitated by one researcher. Interviews were in-depth and lasted up to an hour. To standardise the interview and facilitation techniques, initially the research team carried out joint interviews. Each interview was fully transcribed and kept anonymous.

To achieve methodological triangulation and further explore staff perception, we also looked at the minutes of local Medical Advisory Group meetings, project group meetings and staff consultation meetings on the forthcoming changes.

\section{Data analysis}

The transcripts were systematically analysed for emerging themes by two researchers working independently (G.K. 
and C.D.). Initially themes were coded. Codes included purely descriptive ones arising directly from the transcript, and more interpretive codes. ${ }^{6}$ Any differences

original papers

in interpretation of statements were resolved by discussion with the wider research team until consensus was reached. The coded data were then grouped according to related themes. ${ }^{6}$

The transcripts were read several times to determine how emerging analysis fitted in with the existing data and any new key themes were tested in subsequent interviews.

\section{Results}

Both doctors and non-medical staff discussed similar themes. Generally these were related to their specific area of work rather than their professional background. For example, ward nurses mentioned the positive impact of having fewer ward rounds, whereas CMHT nurses were worried about the potential increase in their workload. However, the implications for psychiatric training were mentioned only by doctors. The ten most frequently cited opinions regarding this change are presented in Box 1.

\section{Opportunities}

Staff commented on the potential benefits of a dedicated in-patient or community doctor. This included quick decision-making, positive risk-taking and improvement in clinical leadership.

'There will be rapid turnover of beds through early discharge and length of stay for patients on the ward will reduce.' (Senior nurse)

It was felt that patient care would improve. There may also be benefit for community staff in the form of respite when a patient is admitted.

'Decrease in number of ward rounds will allow ward staff to spend more time with patients.' (Senior nurse)

'There are benefits of different eyes seeing the same problem ... sometimes you do not realise what is going on with the patient until other people tell you.' (Consultant)

Junior doctors recognised that scope for supervised training will increase. Placements in functional teams will

\section{Box 1. Top ten opinions regarding the change}

Opportunities

- Dedicated doctor time in acute care (ward and crisis team)

- Dedicated doctor time in the community

- Shorter admissions

- Fewer ward rounds and more staff time for patients

- More supervision for junior doctors

Concerns

- Lack of continuity of care

- Differences in opinions between acute care and community teams

- Increase in community team workload

- Lack of consultation and confusion about reasons for change

Solutions

- 'It's communication, communication, communication' provide focused training, essential for development of specialist skills. Trainees will also learn about the importance of communication and good working relationships with colleagues.

'. . learning opportunities will improve . . . but these benefits will be lost if the trusts chose to run the service with locum or staff grade doctors rather than creating rotational training posts.' (Specialty trainee)

\section{Concerns}

The majority of staff were worried about continuity of care. Some did not see this as a new problem.

'Patients will be seen by different teams at different phases of their illness ... the teams will not see the whole picture and will fail to understand the complete nature of the illness.' (Social worker)

'. . . a lot of patients do not need medical follow up after discharge. Of the ones that do only a few will see a consultant and others usually see junior doctors who change frequently anyway.' (Senior nurse)

Concerns were raised about the increase in number of interfaces. In the absence of effective communication there is a risk of some patients 'falling through the net'. Many were worried about possible misunderstanding and disagreement between in-patient and community teams. Issues surrounding Responsible Clinician status and the importance of knowing who is responsible for which decision were also discussed.

'Consultants may find this quite difficult . . . they are quite territorial [laugh] . . . I think they will have a clash of egos.' (Senior nurse)

'Community teams may feel patient is not ready for discharge whilst acute care may think otherwise . . . or disagree regarding need for admission . . . then something bad happens; who takes the responsibility for that? (Specialist registrar)

Staff were worried about de-skilling due to functional nature of work, for example doctors working on in-patient wards may lose touch with issues important in a community setting and vice versa. A potential increase in workload was also anticipated, particularly in the CMHTs. They may not have the capacity to deal with the expected increase in turnover of patients from acute care, which may lead to job dissatisfaction. Many were confused about the reasons for the change and criticised poor communication.

'There is a lack of evidence from research that the functional model is better . . . the change seems to be motivated by political or financial need rather than science.' (Specialty trainee)

$'$. . . heard from other people but there was no formal communication.' (Occupational therapist)

Contrary to some junior doctors who felt training will improve, others raised concerns regarding breadth and depth of training.

'Breadth of experience will be limited . . . the only good thing is it teaches trainees about management of change in the health service, and to know that nothing ever stays the same for more than 30 seconds, which is a skill worth knowing once you are a consultant.' (Consultant) 


\section{How patients might perceive this change}

Staff had several assumptions. Difficulty to engage and confusion regarding the role of different teams were mentioned.

'Patients will be confused as to who is in charge of their care and who they should listen to . . . difference in approach between community and ward consultants may create problems.' (Senior nurse)

It was felt some patients might manage the change better than others, particularly those with frequent admissions. Some might see the benefits of early discharge and being cared for at home.

\begin{abstract}
'. . . some patients may like it; if they do not like their consultant this may be an opportunity for a fresh start . . some patients may not be happy ... this is a change and people are generally resistive about change.' (Specialty trainee)
\end{abstract}

\section{Ideas on how to overcome any difficulties}

There was a range of suggestions on how to overcome potential disagreements and lack of continuity of care in the new system. Central to this was close communication between the acute care and community teams. It was suggested that transfer of care should be clear, jointly agreed care plans should be shared, and teams should attend each others' meetings. Staff thought community and acute care teams should work synergistically and try to complement each other. This is important for effective management of workload and to improve performance. Communication with patients, general practitioners and staff was also thought to be vital to improve understanding of the change.

'Care coordinators should not lose contact when the patient is admitted. They should be involved in ward rounds, care planning meetings and joint home visits with crisis team.' (Senior nurse)

'. . . disagreements should be resolved by discussion keeping the patient's interest in the centre.' (Psychologist)

Secondment of staff to other teams was suggested to enable understanding of the whole system, improve job satisfaction and prevent de-skilling.

'Ward and community staff can be seconded to each other's teams to see how the whole system works and how one team impacts on the other.' (Team leader)

Consistency in approach such as admission thresholds and home treatment criteria were thought to be important. The need for clear guidelines was suggested to prevent confusion regarding role and responsibilities of various teams and their individual members. Several staff raised the importance of formal evaluation of this new system at a later stage.

\section{Discussion}

Our study summarises the perceived impact of this change in service. As more trusts move towards the functional model, it is important to consult staff and address their concerns as part of effective change management. Involving members of the organisation in the change process can be helpful in increasing ownership of a decision or change process, and increasing commitment to it. Communication is also important to overcome the counter-communication, rumours and gossip that are likely to take place. ${ }^{7}$

\section{Impact on quality of care}

Our study pointed out several potential benefits of dedicated ward or community consultants. These include quick decision-making and throughput, improvement in clinical leadership and positive risk management. There is also opportunity for second opinion by default in this system. This is consistent with previous reports on the functional model where quality of patient care improved. $^{8,9}$

Staff raised concerns about the lack of continuity of care. The possibility of disagreements between community and acute care teams and confusion over responsibility was also discussed. The functional model increases interfaces and creates fragmented care. ${ }^{10}$ Having different consultants for in-patient and community care could potentially lead to a disjointed approach. ${ }^{1}$ Our staff suggested improved communication within and between teams as a key to solve this. Joint working and good documentation were suggested as ways to enhance communication.

\section{Impact on staff}

Although staff were generally optimistic and open to change, some were confused about the reasons for this change and worried about how things would work out. There is evidence that any major change in service delivery is likely to cause stress among staff, which can lead to recruitment and retention problems. ${ }^{11}$ This can be minimised by regular discussions between staff and management. ${ }^{11}$ Our staff pointed out the need for more information in advance regarding the reasons and practicalities of this change.

Some raised concerns regarding potential job dissatisfaction and de-skilling in the new model. Secondment of staff to other teams was suggested as one solution. This would also help staff to see the impact of individual teams on the whole service.

\section{Impact on psychiatry training}

Junior doctors pointed out potential benefits to their training. These included more supervision from consultants, training in aspects of acute and community care, competencies in dealing with colleagues, and change management. However, it was recognised that the breadth of clinical experience may be limited unless training in various service settings is ensured. This is also acknowledged by the Psychiatric Trainees Committee of the Royal College of Psychiatrists. ${ }^{12}$ 


\section{Limitations of the study}

original papers
At the time of the study, community staff were aware of further changes in service such as transformation into care pathways later in the year. Staff opinions might have been influenced by this. However, we employed theoretical sampling and did reach saturation of themes, which should increase transferability of the results.

The research team also works in the Trust and was affected by the changes like the participants. In order to minimise observer bias, measures were taken to standardise interviewing techniques and interpretation of statements.

\section{Implications for further research}

It remains to be formally tested whether or not separate hospital and community services provide better psychiatric care than sectorised services. ${ }^{9}$ Parameters that can be examined at a later stage might include number and frequency of re-admissions, bed occupancy, patient and carer satisfaction, number of critical incidents, satisfaction and stress level of ward and community staff, and recruitment and retention rate of staff.

\section{Concluding remarks}

Despite various concerns regarding the change, the need for cohesive working and a patient-centred approach regardless of service design was voiced.

'We have seen changes before, I am sure we can make this work, it doesn't really matter what system we work in . . at the end of the day this is all about team work and caring for patients.' (Senior nurse)

\section{Acknowledgements}

We thank all the staff members who participated in this study. We are also grateful to Dr Jesus Perez, Associate
Clinical Director and Consultant Psychiatrist, and to Maggie Evans, Academic Unit of Primary Health Care, University of Bristol, for their valuable comments on the manuscript.

\section{Declaration of interest}

None.

\section{References}

1 Department of Health. NewWays of Working For Psychiatrists: Enhancing Effective, Personcentred ServicesThrough New Ways of Working in Multidisciplinary and Multi-Agency Contexts. Final Report 'But Not the End of the Story'. Department of Health, 2005.

2 Tyrer P, Al Muderis O, Gulbrandsen D. Distribution of case-load in community mental health teams. Psychiatr Bull 2001; 25: 10-2.

3 Colgan SM. Who wants to be a general psychiatrist? Psychiatr Bull 2002; 26: 3-4.

4 Department of Health. Mental Health Policy Implementation Guide Adult Acute Inpatient Care Provision. Department of Health 2002

5 Department of Health. NewWays of Working for Everyone: Developing and Sustaining a Capable and FlexibleWorkforce. Progress Report. Department of Health, 2007.
6 Miles MB, Huberman AM Qualitative Data Analysis, 2nd edn Sage, 1994

7 Johnson G, Scholes K, Whittington R. Exploring Corporate Strategy: Text and Cases, 7th edn. Financial Times/Prentice Hall, 2004.

8 Harrison J. The work patterns of consultant psychiatrists. Adv PsychiatrTreat 2007; 13: 470-5.

9 Dratcu L, Grandison A, Adkin A. Acutehospital care in inner London: splitting from mental health services in the community. Psychiatr Bull 2003; 27: 83-6.

10 Holloway F. Acute in-patient psychiatry: dedicated consultants if we must but not a specialty. Psychiatr Bull 2006; 30: 402-3.

11 Shah A, DeT. Proposed change in service delivery and stress among nursing staff. Psychiatr Bull1998; 22: $690-2$

12 Malik A, White O, MitchellJ, Henderson P, Oakley C. NewWays ofWorking and psychiatric trainees. Psychiatr Bull 2008; 32: 230-2.

*Golam Khandaker CT2 Specialty Registrar in Adult Psychiatry, Avon and Wiltshire Mental Health Partnership NHS Trust, Grove Road Day Hospital, 12 Grove Road, Redland, Bristol BS6 6UJ, email: golam24@gmail.com, Srinivasarao Cherukuru Staff Grade in Adult Psychiatry, Claire Dibben Specialist Registrar in Adult Psychiatry, Manaan Kar Ray Consultant in Adult Psychiatry, Cambridge and Peterborough NHS FoundationTrust, Peterborough

\section{JOSEPH HAYES, JULIAN MASON, FAY BROWN AND REBECCA MATHER}

\section{Floods in 2007 and older adult services: lessons learnt}

\section{AIMS AND METHOD}

To review the clinical and managerial impact of flooding on a community mental health team (CMHT) for older adults by prospectively recording all flooding related events met by the CMHT.

RESULTS

Of 348 individuals known to the CMHT, 87 lived in flood affected areas. In nine patients symptoms deteriorated. There were two new referrals as a direct consequence of the flooding. Flood effects can be grouped into: new mental illness, management problems, CMHT workload, and secondary benefits.

\section{CLINICAL IMPLICATIONS}

The immediate and delayed problems caused by flooding to the elderly with mental illness and dementia include unmasking cognitive impairment and provoking exacerbations in depressive and anxiety disorders. Personal evacuation plans should be used when moving individuals with marked cognitive impairment to avoid difficulties with identification. Overcrowding of care homes used to temporarily accommodate additional residents can contribute to behavioural changes and psychological symptoms in those with pre-existing dementia. 